\title{
Correction to Semicontinuity for the Local Hilbert Function
}

Juan B. Sancho de Salas

Dpto. de Matemáticas, Universidad de Extremadura, Avda. de Elvas, s/n, E-06071-Badajoz (Spain)

Math. Z. 194, 217-225(1987)

The inequalities $S_{X, y} \leqq \Delta^{r} S_{X, x}$ in Theorems 1.5 and 3.2 are false for $r \geqq 2$. In fact, the given proofs of these theorems show that we have only the weaker inequality

or equivalently,

$$
\Lambda^{-r+1} S_{X, y} \leqq \Delta S_{X, x}
$$

$$
4^{-r} H_{X, y} \leqq H_{X, x}
$$

Therefore, the corrected theorems are:

Theorem 1.5. Let $Y$ be a regular $r$-dimensional closed subscheme of $X=\operatorname{Spec} \mathcal{O}$ defined by a prime ideal $p$ (i.e., $\mathcal{O} / p$ is a regular local ring of dimension $r$ ) and let $y$ be the generic point of $Y$. Then

$$
\Delta^{-r} H_{X, y} \leqq H_{X, x} .
$$

Theorem3.2. Let $Y$ be an excellent $r$-dimensional integral closed subscheme of $X=\operatorname{Spec} \mathcal{O}$, and let $y$ be the generic point of $Y$. Then

$$
\Delta^{-r} H_{X, y} \leqq H_{X, x} .
$$

These changes do not affect the rest of the paper. 